Journal of Gastrointestinal CancerVolume 48, Issue 2, 1 June 2017, Pages 205-207

\title{
Rectal Metastases from Breast Cancer. A Case Report(Article)
}

- Guzmán-Calderón, E. abcdEmail Author

- View Correspondence (jump link)

- ${ }^{a}$ Gastroenterology Unit of the Hospital Nacional Edgardo Rebagliati Martins, Lima, Peru

- 'Universidad Peruana de Ciencias Aplicadas (UPC), Lima, Peru

- Universidad San Martin de Porres (USMP), Lima, Peru

View additional affiliations_

Abstract_View references (10)

[No abstract available]

- ISSN: 19416628

- Source Type: Journal

- Original language: English

- DOI: $10.1007 / \mathrm{s} 12029-016-9827-3$

- Document Type: Article

- Publisher: Humana Press Inc. 